\title{
Study on Multi-Objective Effect Evaluation System of Smart Grid Construction
}

\author{
Yanling Wang, Zhengjie Pan, Haitian Hao, Ziqing Zhou \\ Research Institute of Electric Power Market, North China Electric Power University, Beijing, China \\ Email: wangyanling@ncepu.edu.cn, panzhengjie1992@163.com, haohaitian@sina.com, \\ zhouziqing92@163.com
}

Received January 2015

\begin{abstract}
This paper builds multi-objective effect evaluation indicator system of smart grid construction from five connotations including strong and reliable, clean and green, friendly and interactive, transparent and open, economical and effective, which is embodied in the power generation, transmission, transformation, distribution, consumption, dispatching and information communication platform of smart grid. Taking the construction of smart grid in a certain area of China as an example, this paper uses analytic hierarchy process (AHP) to make an empirical analysis on it, and makes a comprehensive and objective evaluation on its construction effect.
\end{abstract}

\section{Keywords}

\section{Smart Grid, Multi-Objective Effect, Evaluation Indicator System, AHP}

\section{Introduction}

In order to tackle climate change and ensure energy security, countries around the world are increasing emphasis on the development of clean energy and the improvement of energy efficiency at present. As the basis and premise for achieving low-carbon electricity, smart grid technology has developed rapidly in many countries in recent years, and effectively promotes the grid smart [1] [2].

Some countries have put forward appropriate evaluation system of smart grid based on their basic national conditions and development stage of power industry, in order to cooperate with the construction of strong smart grid and reflect its technical characteristics and functional properties comprehensively.

The foreign typical evaluation systems of smart grid mainly contain the smart grid maturity model proposed by IBM [3], the framework system of smart grid assessment drafted by DOE [4], the smart grid construction evaluation indicator of EPRI [5], the smart grid profit evaluation system of EU [6] and so on. It should be noted that the evaluation system of US pays more attention to the safety and reliability of power system because of its obsolete facilities and the hidden dangers against security and stability; the evaluation system of Europe focuses on development and utilization of new energy and low carbon due to the shortage of fossil energy and large specific gravity of new energy generation.

Along with the deepening of smart grid construction in China, domestic scholars are researching and exploring smart grid evaluation hotly, mainly including the establishment of evaluation indicator system, research for 
evaluation model and method [7] [8], etc.

Combined with the target and development direction of smart grid in China, this paper has built multi-objective effect evaluation indicator system of smart grid construction from five connotations including "strong and reliable", "clean and green", "friendly and interactive", "transparent and open", "economical and effective" [9], which can make a comprehensive and objective evaluation on the effect of smart grid construction.

\section{Multi-objective Effect Evaluation Indicator System of Smart Grid Construction}

\subsection{Construction Principle of the Indicator System}

Currently, the World Bank and the national government departments commonly adopt the SMART criterion to select indicators, and the 5 letters of SMART represent specific, measurable, attainable, relevant and trackable. The advantage of this criterion is having a relatively clear standard, which makes evaluation easy.

Considering the characteristics and connotations of smart grid, according to SMART criteria, this paper has proposed the principle of establishing indicator system. 1) Comprehensiveness. Indicators should reflect the action of each construction link as much as possible. 2) Independence. Various indicators in the same level should have clear connotation, mutual independence and cannot overlap each other. 3) Typicality. Indicators should make key points of construction stand out and grasp the main aspect of problem. 4) Measurability. Evaluation indicators of each construction action should have the appropriate standards and use the same standard as unified evaluation dimension.

\subsection{Five Connotations of Smart Grid}

The State Grid Corporation has proposed the development train of thought about unified strong smart grid with Chinese characteristics, which is "one goal, two main lines, three stages, four systems, five connotations, six links". The five connotations are:

1) Strong and reliable, which mean strong grid structure, strong power transmission capacity, safe and reliable power supply ability.

2) Clean and green, which mean promoting development and utilization of renewable energy, reducing energy consumption and pollutant emission, and improving the proportion of clean energy in final energy consumption.

3) Friendly and interactive, which mean flexible adjustment of power grid operation mode, easy for various types of power and users accessing or exiting the grid, promoting the generation companies and users to actively participate in grid operation regulation.

4) Transparent and open, which mean the transparent and shared information of power grid, power sources and users, and open grid without discrimination.

5) Economical and effective, which mean improving the operation and transportation efficiency of power grid, reducing operating costs, promoting the efficient utilization of energy resources and electric power assets.

According to these five connotations above, the effect evaluation indicator system of smart grid construction extracts effect indicators from each construction link of smart grid, which can embody the smart grid characteristics scientifically and comprehensively.

\subsection{Multi-Objective Effect Evaluation Indicator System}

The smart grid has five connotations including "strong and reliable”, "clean and green”, "friendly and interactive”, "transparent and open", "economical and effective", which are embodied in the power generation, transmission, transformation, distribution, consumption, dispatching and information communication platform of smart grid. Build multi-objective effect evaluation indicator system of smart grid construction based on these connotations, as shown in Table 1.

\section{Effect Evaluation Model Based on AHP}

\subsection{Determine the Weight of Indicators by AHP}

The multi-objective effect evaluation indicator system of smart grid construction has obvious hierarchical structure, as shown in Figure 1. Because there are more species and quantity of indicator, and the indicator system is large, it is suitable to use AHP to solve the problem of reasonable weight of indicator and then evaluate the 
Table 1. Multi-objective effect evaluation indicator system.

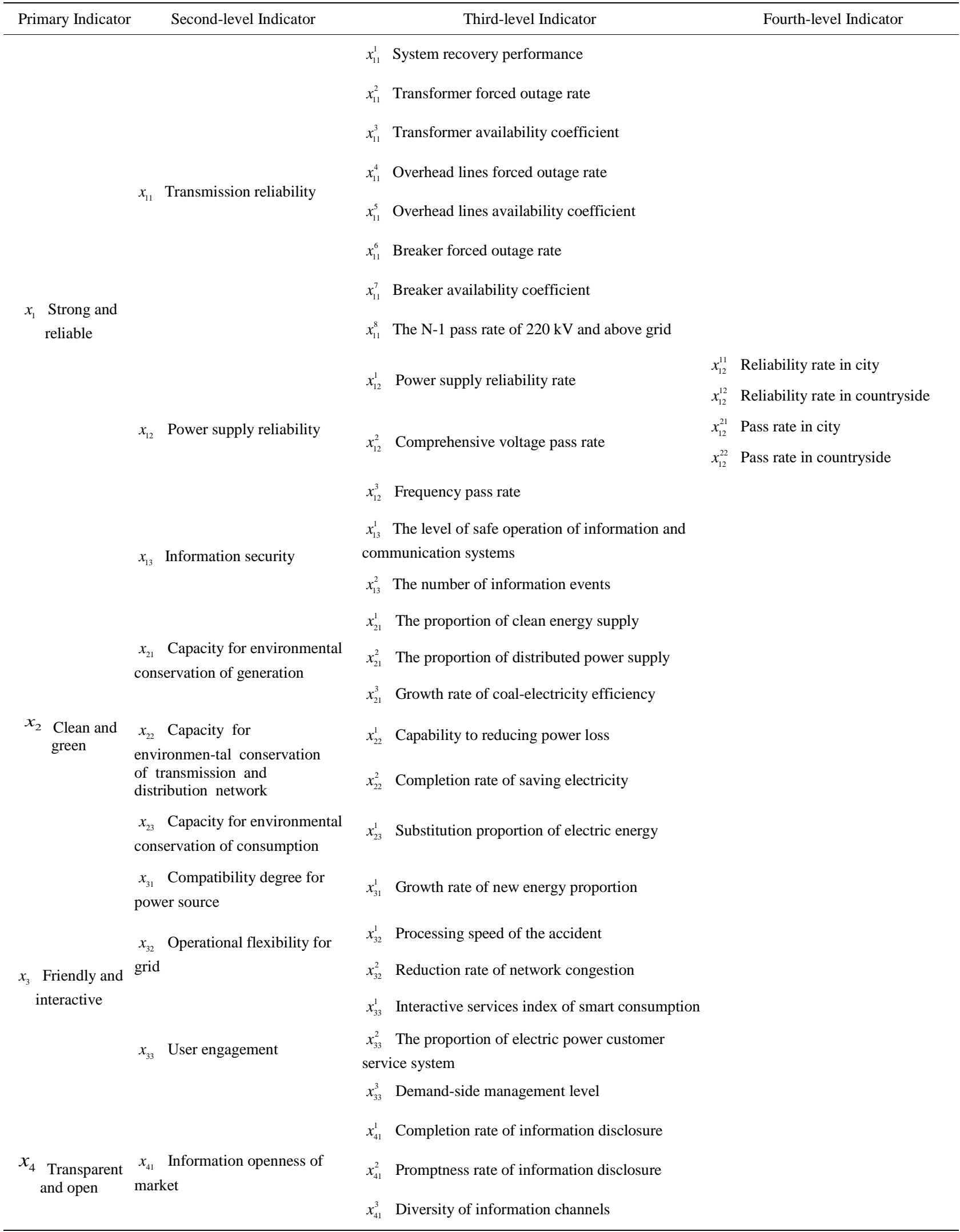




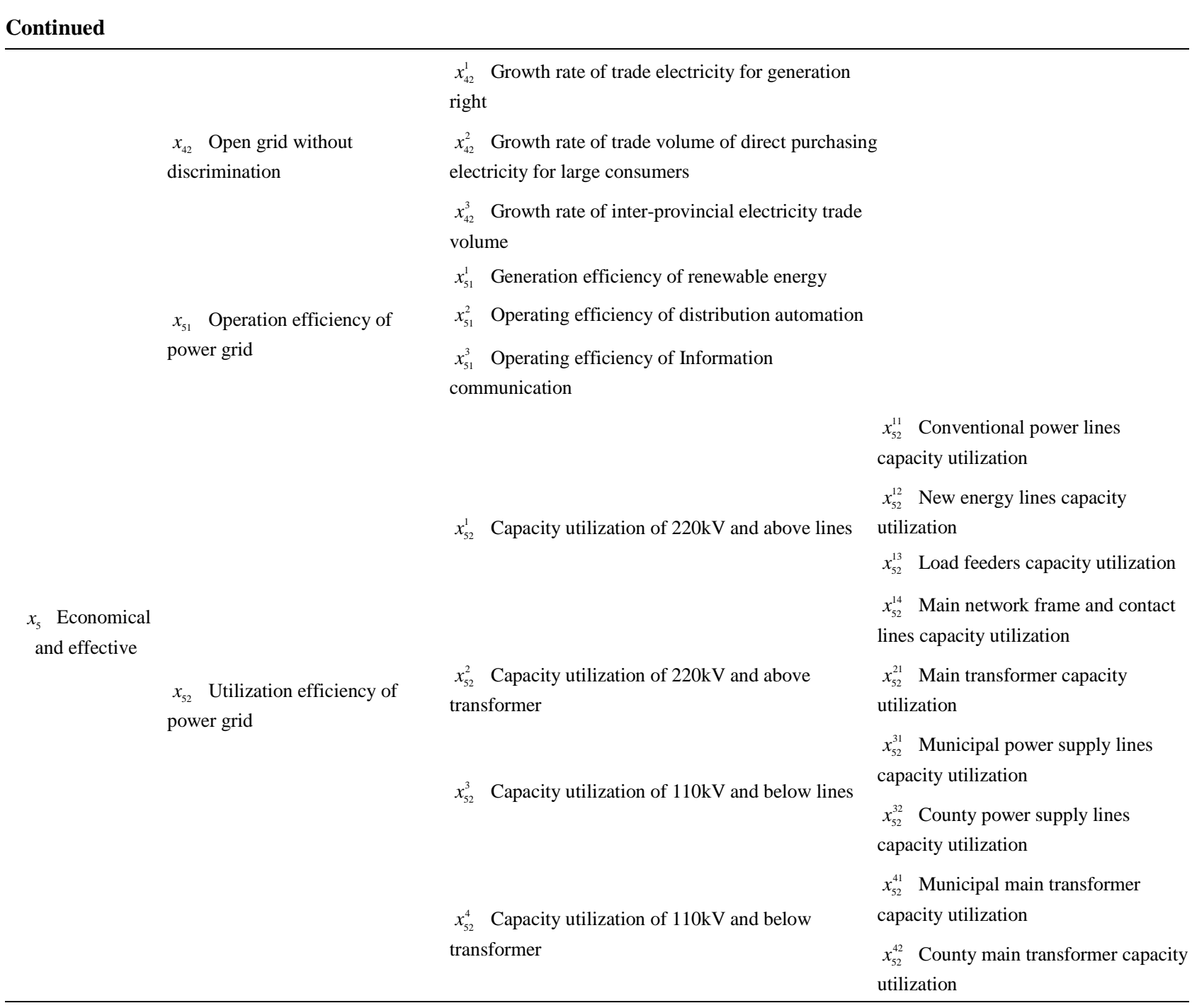

construction effect of smart grid. Specific steps are as follows:

1) Build the hierarchic analysis structure. The Figure 1 just shows typical AHP model structure.

2) Construct the judgment matrix. The judgment matrix compares the relative importance degree of evaluation indicators at the same level which are associated with the indicator in upper level.

3) Order the single hierarchical. Calculate relative importance of a factor in one hierarchy to a factor in the upper hierarchy, which is called single hierarchical ordering. It can come down to calculating the largest eigenvalues and eigenvectors of the judgment matrix.

4) Test the consistency of judgment matrix. The indicator weight assignment is successful if the result of single hierarchical ordering has an ideal consistency; otherwise, the values of element in judgment matrix need to be adjusted.

\subsection{Classify the Indicator Evaluation Grade}

Set four evaluation grades for effect indicators, give each grade the corresponding score interval, and then we can classify the effect level of smart grid construction, as shown in Table 2.

\subsection{Calculate the Evaluation Result}

Calculate each indicator score by using weighted means method, the evaluation results of five primary indicators 


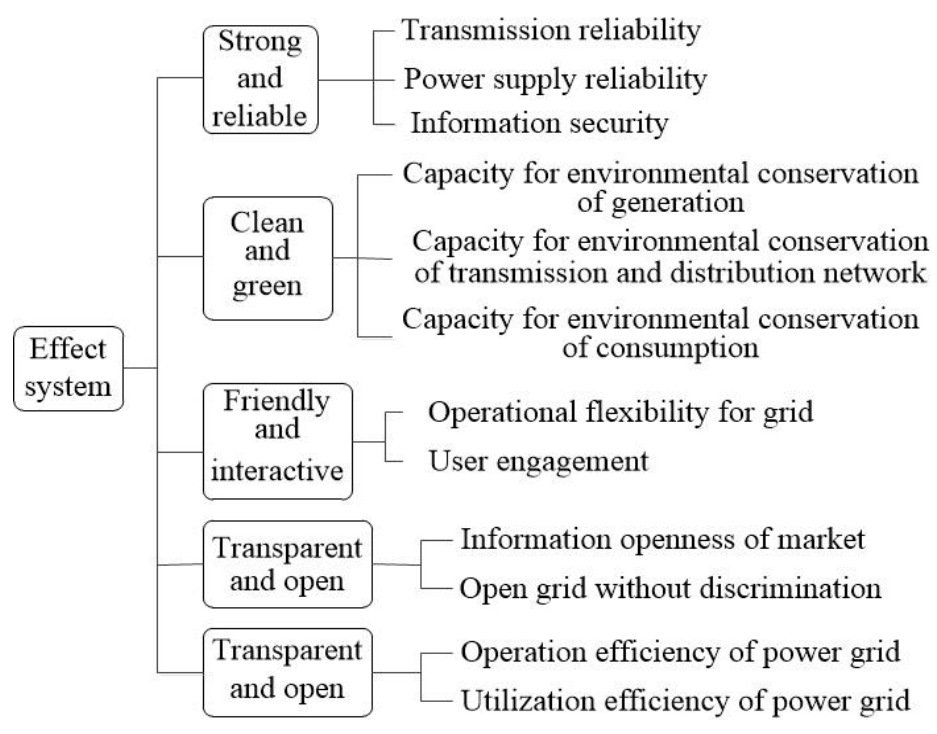

Figure 1. Hierarchical chart of multi-objective effect evaluation system.

Table 2. Evaluation grade and score interval of effect.

\begin{tabular}{ccc}
\hline No. & Indicator Evaluation Grade & Score Interval \\
\hline A & Unqualified & $0-59$ \\
B & Qualified & $60-69$ \\
C & Good & $70-84$ \\
D & Excellent & $85-100$ \\
\hline
\end{tabular}

in multi-objective effect evaluation indicator system can be obtained according to the score interval and evaluation grade in Table 2, as shown in Table 3.

Finally, we can judge the overall level of smart grid construction effect by using the weighted means method to obtain total score according to the scores of five primary indicators.

\section{Example Analysis}

Use the method in this paper to evaluate construction effect of smart grid in one region.

\subsection{Determine the Indicator Weight and Score}

Determine the indicator weight by using AHP, and let experts give the corresponding scores on the basis of actual data of smart grid. The indicator weight and score are as shown in Figure 2.

\subsection{Calculate the Evaluation Result}

Use weighted means method to calculate scores of the five primary indicators including "strong and reliable", "clean and green", "friendly and interactive", "transparent and open", "economical and effective", and finally obtain the total score of effect. Then judge the grade of construction effect according to Table 2, as shown in

\section{Table 4.}

Finally, we can judge the overall level of smart grid construction effect by using the weighted means method to obtain total score according to the scores of five primary indicators.

We can see from Table 4 that the overall effect of the smart grid construction shows a good level. "Strong and reliable" and "friendly and interactive" are excellent, while "clean and green", "transparent and open" and "economical and effective" are good. Weak part of smart grid construction in one region can be found out through in-depth analysis of basic indicators. For example, the quality of power supply in rural areas is relatively 
Table 3. Evaluation result of smart grid construction effect example.

\begin{tabular}{ccc}
\hline Indicator & Score & Evaluation Grade \\
\hline Strong and reliable & 90 & Excellent \\
Clean and green & 84 & Good \\
Friendly and interactive & 92 & Excellent \\
Transparent and open & 81 & Good \\
Economical and effective & 72 & Good \\
\hline
\end{tabular}

Table 4. Evaluation result of smart grid construction effect.

\begin{tabular}{ccc}
\hline Indicator & Score & Evaluation Grade \\
\hline Strong and reliable & 94.02 & Excellent \\
Clean and green & 82.14 & Good \\
Friendly and interactive & 87.17 & Excellent \\
Transparent and open & 81.43 & Good \\
Economical and effective & 71.05 & Good \\
Total effect & 83.16 & Good \\
\hline
\end{tabular}

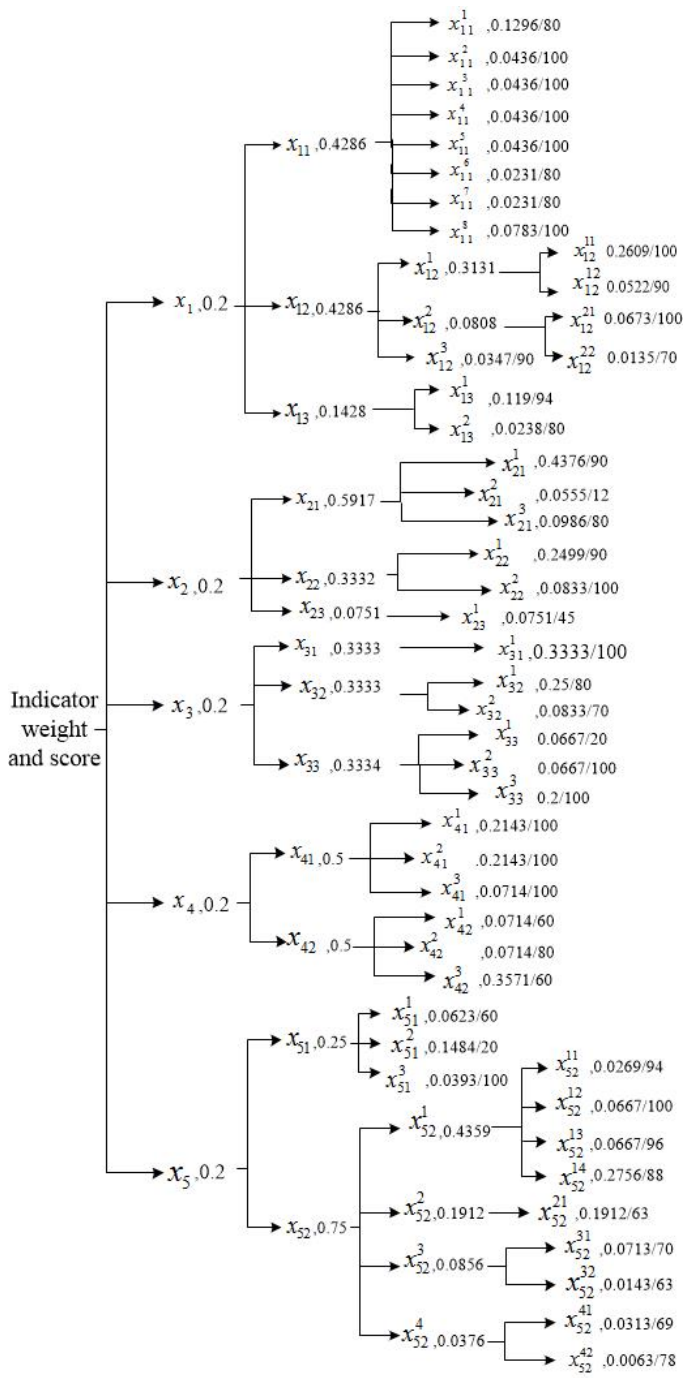

Figure 2. Indicator weight and score. Note: “x, Digital 1/Digital 2” represent “indicator, weight/value”. 
poor, distributed power develops slowly, the level of environmental protection on power demand side is low, various electricity trades increase slow, utilization efficiency of grid a little low, etc. Therefore, appropriate measures should be taken to continuously strengthen and improve the construction work of smart grid, such as reinforcing the construction and renovation of rural power grid, rational development of distributed power, actively promoting the strategy of electric energy substitution, further improving the trading rules and trading system functions of power market, further optimizing the grid structure.

\section{Conclusions}

This paper constructs multi-objective effect evaluation indicator system around five connotations including strong and reliable, clean and green, friendly and interactive, transparent and open, economical and effective; and builds the evaluation model based on AHP. Through the evaluation and analysis of smart grid construction in one region of China, it is found that "clean and green", "transparent and open" and "economical and effective" get low scores. There is a need to improve the construction work of smart grid for its weak part, thereby guaranteeing the scientifically and orderly development of the construction work.

The case in this paper shows that the evaluation system can objectively evaluate the construction effect of smart grid in the round, quickly and accurately identify the deficiencies in smart grid construction, so that it can help to grasp the development direction of smart grid construction and provide reference for the planning and construction of smart grid.

\section{References}

[1] Zheng, J. (2011) The Review of the Smart Grid Development Both at Home and Abroad Shanghai Electric Power, No. 3, 256-259.

[2] Bala, S.K., Babu, B.C. and Bala, S. (2012) A Review on Development of Smart Grid Technology in India and Its Future Perspectives. Proceedings of 2012 Students Conference on Engineering and Systems (SCES 2012), Uttar Pradesh, 16-18 March 2012, 1-6. http://dx.doi.org/10.1109/SCES.2012.6199016

[3] IBM Corporation (2009) IBM End-to-End Security for Smart Grids. IBM Corporation, New York.

[4] US Department of Energy (2009) Smart Grid System Report. U.S. Department of Energy, New York.

[5] The Electric Power Research Institute Inc., EPRI (2010) Methodological Approach for Estimating the Benefits and Costs of Smart Grid Demonstration Projects. The Electric Power Research Institute Inc., Palo Alto.

[6] European Smart Grids Technology Platform (2010) Vision and Strategy for Europe’s Electricity Networks of the Future. European Commission, Brussels.

[7] Wang, J.-M. and Shi, T. (2012) Construction of Evaluation Index System for Smart Grid. East China Electric Power, 40, 193-197.

[8] Sun, Q., Ge, X.-B., Liu, L., Xu, X., Zhang, Y.-B., Zeng, Y., et al. (2011) Review of Smart Grid Comprehensive Assessment Systems. Proceedings of the CSU-EPSA, 23, 105-109.

[9] Baidu Encyclopedia, Smart Grid. http://baike.baidu.com/view/2222513.htm?fr=aladdin 